\title{
In silico search for multi-target therapies for osteoarthritis based on 10 common Huoxue Huayu herbs and potential applications to other diseases
}

\author{
CHUN-SONG ZHENG ${ }^{1}$, ZHI-QIANG ZHUANG ${ }^{2}$, XIAO-JIE XU ${ }^{1,3}$, JIN-XIA YE $^{1}$, HONG-ZHI YE ${ }^{1}$, \\ XI-HAI LI ${ }^{1}$, GUANG-WEN WU ${ }^{1}$, HUI-FENG XU ${ }^{1}$ and XIAN-XIANG LIU ${ }^{1}$ \\ ${ }^{1}$ Academy of Integrative Medicine, Fujian University of Traditional Chinese Medicine, Fujian 350122; \\ ${ }^{2}$ Affiliated Zhangzhou Hospital of Traditional Chinese Medicine, Fujian University of Traditional Chinese Medicine, \\ Fujian 363000; ${ }^{3}$ College of Chemistry and Molecular Engineering, Peking University, Beijing 100871, P.R. China
}

Received May 28, 2013; Accepted January 16, 2014

DOI: $10.3892 / \mathrm{mmr} .2014 .1914$

\begin{abstract}
Huoxue Huayu (HXHY) has been widely used in traditional Chinese medicine (TCM) as a key therapeutic principle for osteoarthritis (OA), and related herbs have been widely prescribed to treat $\mathrm{OA}$ in the clinic. The aims of the present study were to explore a multi-target therapy for OA using 10 common HXHY herbs and to investigate their potential applications for treatment of other diseases. A novel computational simulation approach that integrates chemical structure, ligand clusters, chemical space and drug-likeness evaluations, as well as docking and network analysis, was used to investigate the properties and effects of the herbs. The compounds contained in the studied HXHY herbs were divided into 10 clusters. Comparison of the chemical properties of these compounds to those of other compounds described in the DrugBank database indicated that the properties of the former are more diverse than those of the latter and that most of the HXHY-derived compounds do not violate the 'Lipinski's rule of five'. Docking analysis allowed for the identification of 39 potential bioactive compounds from HXHY herbs and 11 potential targets for these compounds. The identified targets were closely associated with 49 diseases, including neoplasms, musculoskeletal, nervous
\end{abstract}

Correspondence to: Professor Xian-Xiang Liu, Academy of Integrative Medicine, Fujian University of Traditional Chinese Medicine, 1 Huatuo Road, Minhou Shangjie, Fuzhou, Fujian 350122, P.R. China

E-mail: liuxianxiang@163.com

Abbreviations: TCM, traditional Chinese medicine; HXHY, Huoxue Huayu; OA, osteoarthritis; ADAMTS-5, aggrecanase-2; MMP, matrix metalloproteinase; TNF- $\alpha$, tumor necrosis factor- $\alpha$; iNOS, inducible nitric oxide synthase; PPAR $\gamma$, peroxisome proliferator activated receptor $\gamma$; PDE, phosphodiesterase; L-T-D network, ligand-target-disease network; TTD, therapeutic target database

Key words: osteoarthritis, multi-target therapy, Huoxue Huayu herbs, computational simulation system and cardiovascular diseases. Ligand-target (L-T) and ligand-target-disease (L-T-D) networks were constructed in order to further elucidate the pharmacological effects of the herbs. Our findings suggest that a number of compounds from HXHY herbs are promising candidates for mult-target therapeutic application in OA and may exert diverse pharmacological effects, affecting additional diseases besides OA.

\section{Introduction}

Osteoarthritis (OA) is a degenerative joint disease, which causes chronic pain and functional restrictions in the affected joints (1). At present, there is no effective treatment for reversing or preventing its onset. Non-steroidal anti-inflammatory drugs (NSAIDs) are mainly used for treating OA, particularly in the early stages of the disease, but these drugs are often associated with clinically adverse effects (2). Furthermore, the social and economic cost related to OA remains particularly high (3). Therefore, ongoing research attempts to develop improved therapeutic strategies for OA.

Several lines of evidence suggest that treatment that aims at a number of targets at once may be more effective against complex diseases such as OA $(4,5)$. On the other hand, traditional Chinese medicine (TCM), a complex system employing multiple components and targets, has been recognized in Western countries as a popular complementary and alternative medicinal approach. TCM has been used in the treatment of $\mathrm{OA}$, and often has fewer side-effects than those reported for NSAIDs (6-8). Those reports indicate that TCM may provide a novel promising strategy for treatment of OA.

Huoxue Huayu (HXHY) has been widely used in TCM as a key therapeutical principle for OA in China $(9,10)$. Ten common HXHY herbs, Ligusticum chuanxiong, Salvia miltiorrhiza, Strychnos nux-vomica, Persicae semen, Corydalis yanhusuo, Drynaria fortunei, Commiphora myrrha, Carthamus tinctorius, Boswellia carterii and Achyranthes bidentata, have been reported to play an important role in the treatment of OA (9-13). However, the mechanisms underlying the effects of these herbs are poorly studied. Therefore, in the present study, we investigated the pharmacology and effectiveness of 
these herbs using an integrative model, combining chemical structure, ligand clusters, chemical space and drug-likeness evaluations, as well as docking screening and network analysis. Our study aimed to contribute in the elucidation of the mechanisms underlying HXHY herb effects and in the long term, in developing strategies for OA treatment.

\section{Materials and methods}

Chemical structures and clustering. The chemical structures of compounds contained in the 10 aforementioned HXHY herbs were downloaded from the Chinese Herbal Drug Database (14). Following duplicate exclusion, a total of 208 chemical structures were retained. They were converted into three-dimensional structures and energy optimizations were performed using the Discovery Studio 2.0 (DS 2.0) software (Accelrys, Inc., San Diego, CA, USA) based on the Merck Molecular Force Field (MMFF). The Cluster Ligands protocol was then used to cluster the compounds from HXHY herbs. In addition, 96 drug/drug-like compounds in association with OA disease were collected from the DrugBank database (15) and were optimized based on the MMFF.

Chemical space mapping and drug-likeness prediction. Chemical space was estimated by calculating a given set of descriptors for each molecule and using these values as coordinates in the multi-dimensional space (16). In the present study, 34 common descriptors were used to estimate the chemical space for compounds from HXHY herbs and DrugBank using the Calculate Molecular Properties protocol available in the Quantitative Structure-Activity Relationship module of DS 2.0 (17). Then, principal component analysis (PCA) was performed to map the distribution of compounds in the chemical space. In addition, calculations of the 'Lipinski's rule of five' and of chemical space were used to evaluate whether the tested herbal compounds were drug-like (18).

Docking simulations. The crystal structures of the protein-ligand complexes of 11 protein targets related to OA (Table I) were retrieved from the Research Collaboratory for Structural Bioinformatics Protein Data Bank (RCSB PDB; www.rcsb.org) (19-21). Crystallographic water molecules were removed and hydrogen atoms were added in the file. The inhibitor reported on the PDB file was used to define the active site. The compounds from HXHY herbs were docked to these targets using the LigandFit module of DS 2.0. The docking was performed by generating sets of different compound conformations using a Monte Carlo algorithm, and matching these conformations to the binding site partitions (22). All 208 docked structures were thus sorted according to their DockScore. The DockScore of each target and its original inhibitor was used as the cut-off value, so that the targets and compounds with the higher DockScore were selected as potential targets and bioactive compounds.

Network construction and analysis. The procedure for network construction was the following: First, the 'ligand-target network' (L-T network) was established by connecting the predicted targets and bioactive compounds. Then, the disease list associated with the 11 targets was
Table I. Eleven protein targets related to osteoarthritis.

\begin{tabular}{lll}
\hline Protein & \multicolumn{1}{c}{ Full name } & PDB code \\
\hline MMP-12 & Matrix metalloproteinase-12 & 3EHX \\
MMP-8 & Matrix metalloproteinase-8 & 1ZP5 \\
MMP-9 & Matrix metalloproteinase-9 & 1GKC \\
MMP-3 & Matrix metalloproteinase-3 & 1HY7 \\
MMP-2 & Matrix metalloproteinase-2 & 1HOV \\
ADAMTS-5 & Aggrecanase-2 & 2RJQ \\
PPAR $\gamma$ & Peroxisome proliferator-activated & 2VSR \\
& receptor $\gamma$ & \\
PDE-4a & Phosphodiesterase-4a & $3 \mathrm{I} 8 \mathrm{~V}$ \\
PDE-4d & Phosphodiesterase-4d & $3 \mathrm{AIK}$ \\
iNOS & Inducible nitric oxide synthase & 2ORO \\
TNF- $\alpha$ & Tumor necrosis factor- $\alpha$ & 2AZ5
\end{tabular}

PDB, Protein Data Bank (Research Collaboratory for Structural Bioinformatics member).

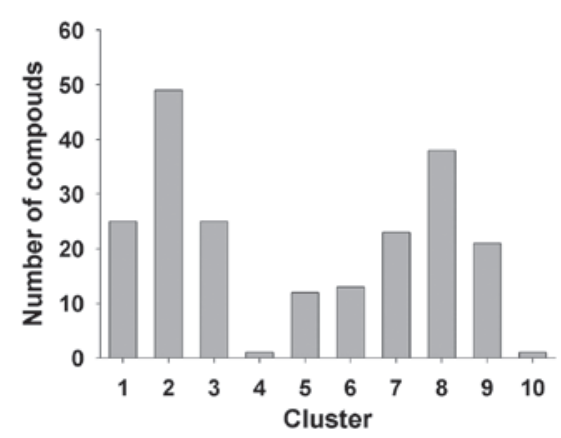

Figure 1. Distribution of compounds from Huoxue Huayu herbs based on clustering analysis.

obtained from the Therapeutic Target Database (23) and the diseases were also classified into different groups using the medical subject headings terms (http://www.nlm.nih.gov/). A ligand-target-disease (L-T-D) network was constructed by connecting previously mentioned proteins to any associated diseases, and the diseases to different groups based on the L-T network. Cytoscape 2.8.3 analysis was carried out to construct these networks (24). In the networks, the compounds, targets and diseases are represented as nodes, and the edges between nodes represent intermolecular interactions. All data were analyzed using Cytoscape plugins (25).

\section{Results}

Chemical diversity and drug-likeness prediction. Compounds from HXHY herbs were subdivided into 10 clusters by employing the default settings of the Cluster Ligands protocol (Fig. 1). PCA revealed considerable dispersion in the chemical space distribution of the compounds from HXHY herbs (Fig. 2A). Some of compounds from HXHY herbs occupied similar chemical space with compounds from 
Table II. The mean, minimum (Min) and maximum (Max) values of molecular descriptors for the compounds from 10 Huoxue Huayu herbs.

\begin{tabular}{lccc}
\hline Name & Mean & Min & Max \\
\hline C count & 18.66 & 3 & 39 \\
H count & 23.05 & 6 & 60 \\
O count & 4.06 & 0 & 16 \\
ALogP & 3 & -7.46 & 13.6 \\
Apol & $12,174.84$ & $2,113.82$ & $27,958.8$ \\
Molecular weight & 319.15 & 59.11 & 742.92 \\
No. of atoms & 23.2 & 4 & 53 \\
No. of rotatable bonds & 3.79 & 0 & 26 \\
No. of rings & 3.27 & 0 & 7 \\
No. of H acceptors & 4.22 & 0 & 16 \\
No. of H donors & 1.69 & 0 & 15 \\
Wiener & $1,497.58$ & 9 & 11,322 \\
Zagreb & 126.63 & 12 & 274 \\
Molecular volume & 220.79 & 55.56 & 444.52 \\
Molecular surface area & 312.02 & 91.4 & 692.23 \\
\hline
\end{tabular}

Table III. Parameters of the ligand-target (L-T) network.

\begin{tabular}{lc}
\hline Parameter & $\begin{array}{c}\text { HXHY L-T } \\
\text { network value }\end{array}$ \\
\hline No. of nodes & 50 \\
No. of edges & 138 \\
Network density & 0.113 \\
Network heterogeneity & 0.99 \\
Isolated nodes & 0 \\
No. of self-loops & 0 \\
Multi-edge node pairs & 0 \\
Network centralization & 0.435 \\
No. of shortest paths (\%) & $2,450(100)$ \\
Characteristic path length & 2.52 \\
Average no. of neighbors & 5.52 \\
\hline
\end{tabular}

DrugBank (Fig. 2B). Furthermore, evaluation of the drug-like properties (Table II) showed that $95.19 \%$ of the compounds had a molecular weight $<500,92.79 \%$ had $<10$ H-bond acceptors, $90.38 \%$ had $<5 \mathrm{H}$-bond donors and $82.69 \%$ had ALogP $<5$. These results demonstrated that the compounds from HXHY herbs possess chemical diversity and drug-likeness.

Prediction of potential targets and bioactive compounds. A virtual screening approach was adopted for compounds with the potential to inhibit protein targets related to OA. Among the 39 compounds screened, 28 were predicted to interact with more than one target.

The ligand-target space prediction. Potential ligand-target interactions were described in the HXHY L-T network (Fig. 3). Table III lists the values for a few simple parameters of the L-T
Table IV. Top 10 compounds exhibiting the highest degrees of connectivity in the ligand-target network.

\begin{tabular}{rllc}
\hline Index & Known & \multicolumn{1}{c}{ Chemical name } & Degree \\
\hline 89 & No & Salvianolic acid C & 9 \\
124 & Yes & Safflor yellow A & 8 \\
78 & No & Monomethyl lithospermate & 8 \\
113 & No & 6-Hydroxykaempferol-7-O-glucoside & 7 \\
88 & No & Salvianolic acid A & 7 \\
103 & Yes & Naringin & 7 \\
187 & Yes & Coptisine & 6 \\
26 & No & Folic acid & 6 \\
55 & Yes & Danshensuan B & 6 \\
86 & Yes & Rosmarinic acid & 5 \\
\hline
\end{tabular}

The term index represents individual compounds from the Huoxue Huayu herbs.
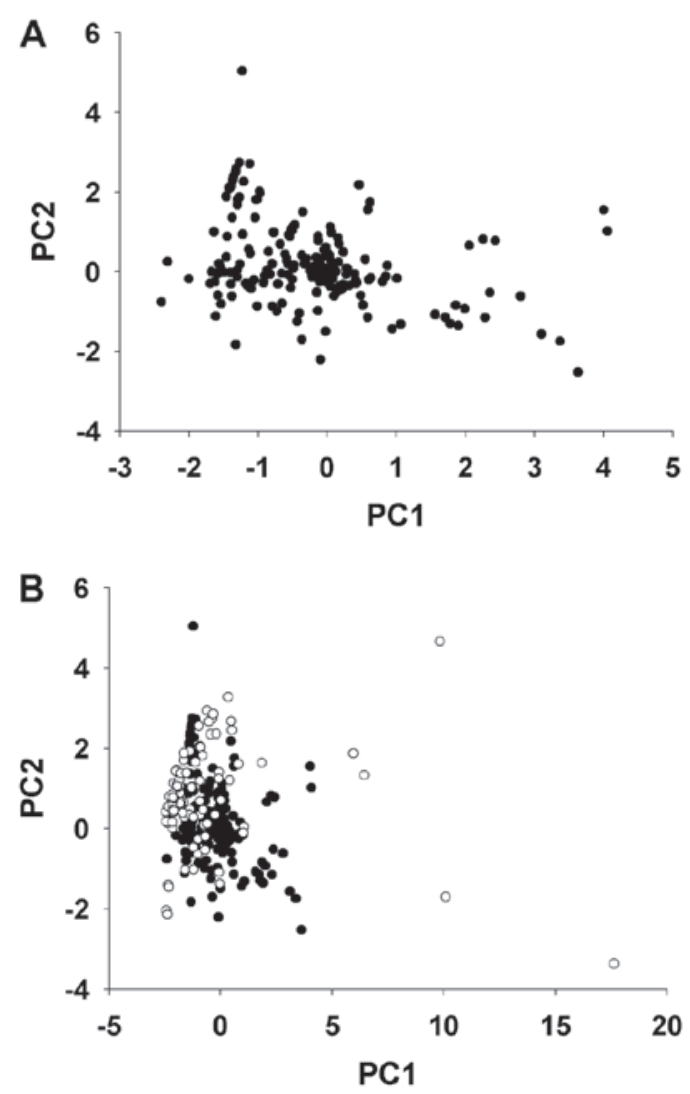

Figure 2. Plot of the first two principal components of compounds from Huoxue Huayu herbs (black circles) and the DrugBank (white circles). (A) Considerable dispersion in the chemical space distribution of the compounds from HXHY herbs. (B) Some of the compounds from HXHY herbs occupied similar chemical space with compounds from DrugBank. PC1, first principal component; $\mathrm{PC} 2$, second principal component.

network. The L-T network contains 50 nodes (39 ligands and 11 potential targets) and 138 edges. The network centralization and network heterogeneity were estimated at 0.435 and 0.99 , respectively. This indicates that a few nodes are more central than others in the network. The analysis of the degree of connec- 
Table V. The 49 diseases related to the 11 targets.

\begin{tabular}{|c|c|}
\hline Index & Disease \\
\hline D1 & Abscess \\
\hline D2 & $\begin{array}{l}\text { Adrenocorticotrophic hormone-secreting } \\
\text { pituitary tumors }\end{array}$ \\
\hline D3 & Advanced lung cancer \\
\hline D4 & Asthma \\
\hline D5 & Atherosclerosis \\
\hline D6 & Atopic dermatitis \\
\hline D7 & Autoimmune diseases \\
\hline D8 & Behcet's disease \\
\hline D9 & Bladder cancer \\
\hline D10 & Brain Cancer \\
\hline D11 & Breast cancer \\
\hline D12 & Chronic fatigue syndrome \\
\hline D13 & Congestive heart failure \\
\hline D14 & Crohn's disease \\
\hline D15 & Diabetes mellitus \\
\hline D16 & Duchenne muscular dystrophy \\
\hline D17 & Emphysema \\
\hline D18 & Guillain-Barre syndrome \\
\hline D19 & Hepatocellular carcinoma \\
\hline D20 & Hormone-refractory prostate cancer \\
\hline D21 & Hyperimmunoglobulinemia D \\
\hline $\mathrm{D} 22$ & Inflammation \\
\hline D23 & Inflammatory bowel disease \\
\hline D24 & Insulin resistance \\
\hline D25 & Ischemia reperfusion injuries \\
\hline D26 & Ischemic heart disease \\
\hline $\mathrm{D} 27$ & Kaposi's sarcoma \\
\hline D28 & Lung cancer \\
\hline D29 & Multiple sclerosis \\
\hline D30 & Muscle atrophy \\
\hline D31 & Myocardial infarction \\
\hline D32 & Noninsulin-dependent diabetes mellitus \\
\hline D33 & Non-small cell lung cancer \\
\hline D34 & Obesity \\
\hline D35 & Ulcerative colitis \\
\hline D36 & Osteoarthritis \\
\hline D37 & Osteoporosis \\
\hline D38 & Ovarian cancer \\
\hline D39 & Pancreatic cancer \\
\hline D40 & Prostate cancer \\
\hline D41 & Psoriasis \\
\hline D42 & Renal cell carcinoma \\
\hline D43 & Renal interstitial fibrosis \\
\hline D44 & Restenosis \\
\hline D45 & Rheumatic diseases \\
\hline D46 & Rheumatoid arthritis \\
\hline D47 & Smooth muscle hyperplasia \\
\hline D48 & Testicular cancer \\
\hline D49 & Thyroid follicular carcinoma \\
\hline
\end{tabular}

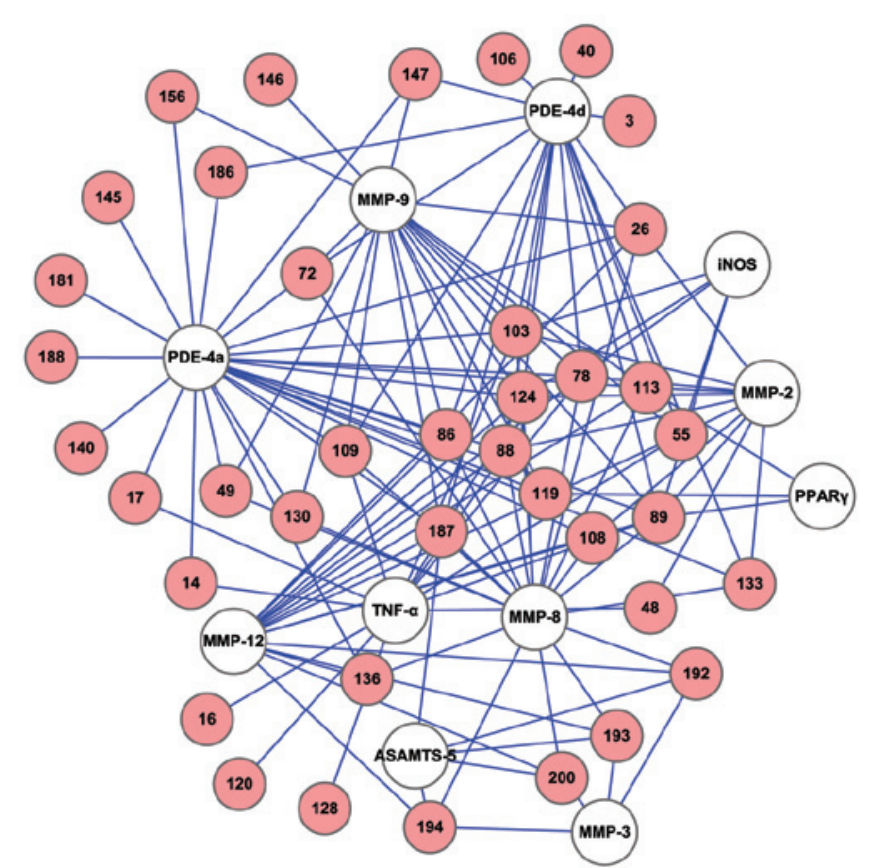

Figure 3. Huoxue Huayu (HXHY) ligand-target network. The pink and white nodes (circles) represent compounds (ligands) from HXHY herbs and the targets related to osteoarthritis, respectively. The edges represent interactions between nodes.

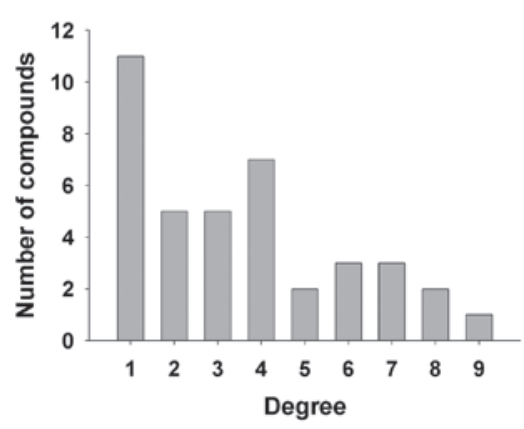

Figure 4. Distribution of compounds for different degrees of connectivity in the Huoxue Huayu ligand-target network.

tivity for the compounds in the HXHY L-T network is presented in Fig. 4 and the chemical names of the top 10 compounds with regards to the degree of connectivity are shown in Table IV.

Associations of HXHY herb compounds with other diseases. The predicted targets were associated with a total of 49 diseases (Table V). According to the Medical Subject Headings controlled vocabulary (http://www.nlm.nih.gov/), these diseases are classified into 19 groups. The L-T-D network was also constructed to explore the interactions and identify potential roles for the compounds of HXHY herbs (Fig. 5). Overall, these results indicate that HXHY herbs may act beneficially in a range of distinct diseases.

\section{Discussion}

The pathogenesis of OA appears to be the result of multiple abnormalities including those in protease and cytokine activities (26). A single drug is most probably insufficient for OA 


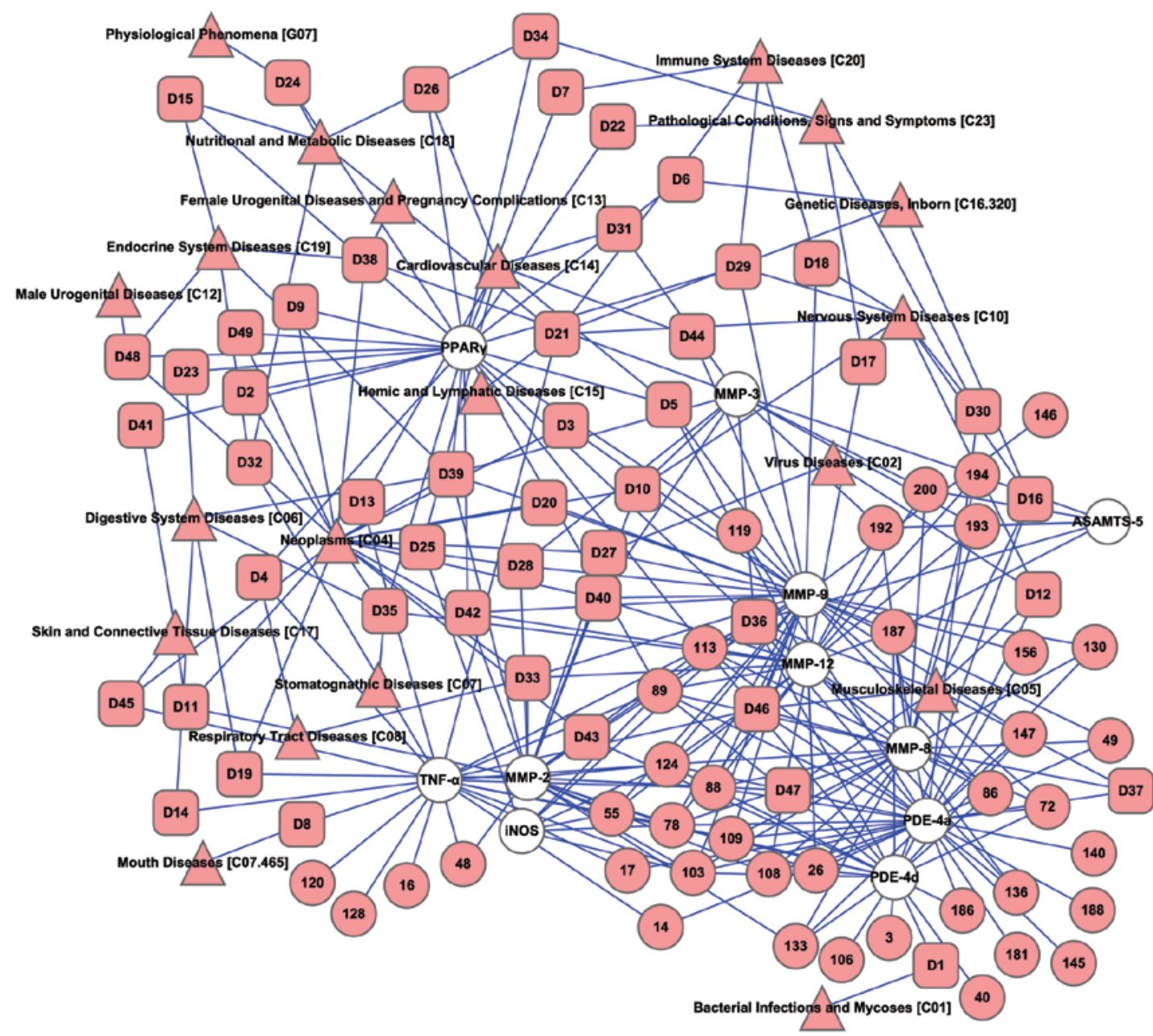

Figure 5. The ligand-target-disease network. The white and pink nodes (circles) represent the targets and the ligands (compounds) from Huoxue Huayu herbs, respectively, the rectangles represent the diseases related to the 11 targets and the triangles represent the disease classifications according to the Medical Subject Headings classification system.

therapy. It was previously hypothesized that a complex disease with multi-factorial pathophysiology can be more effectively treated through the use of a multi-drug mixture as compared to a single drug (27). Therefore, multi-target drug treatment is a promising approach for clinical OA therapy.

In the present study, we used docking simulations to identify the protein targets of HXHY herbs, based on the chemical diversity (Fig. 1) and drug-likeness (Fig. 2) of compounds (ligands) contained in these herbs. Twenty-eight compounds were predicted to bind to more than one protein. Evidence suggests that such compounds, also known as promiscuous drugs, may present several benefits (28). It is foreseeable that a 'one-drug-multiple-targets' therapeutic strategy is to be adopted for treatment of OA in the future. In addition, we identified 11 compounds predicted to bind to only one target. These compounds could be considerably potent when combined, with different combinations selectively targeting different multiple targets. These findings suggest that the studied herbs may be useful sources of both promiscuous drugs and combination drugs that can be used in combination therapies.

To elucidate the relationships between the active compounds and their targets, the L-T network was constructed by connecting ligands to the corresponding targets (Fig. 3). The average number of potential targets per compound was 3.5. Generally, the compounds with higher degree of connectivity are more potent pharmacologically (29). A few of the compounds identified here as having a high-degree of connectivity (Table IV) have been reported in the literature (30-32). These results overall suggest that HXHY herbs may simultaneously target multiple proteins.

Considering the distinct effects and applications of HXHY herbs in the clinic (33-35), we constructed the L-T-D network (Fig. 5) to link the 11 targets and the related diseases to gain a global understanding of diseases associated with the compounds of the L-T network. It is believed that compounds targeting the same protein that is associated with different diseases may be beneficial in different diseases (36). A total of 49 diseases showed associations with the compounds of HXHY herbs (Table V). This finding suggests that HXHY herbs demonstrate considerable potency for musculoskeletal diseases, as well as neoplasms, the nervous system, cardiovascular, nutritional and metabolic diseases. For instance, matrix metalloproteinase-12, which was predicted as the target of coptisine (Table IV), is associated with diverse diseases, including musculoskeletal, cardiovascular and digestive system diseases (23). Previous studies have provided evidence that coptisine selectively prevents vascular smooth muscle cell proliferation at low concentrations and exerts a cardioprotective effect through its antioxidative properties and inhibition of the RhoA/Rho 
kinase pathway in rats with isoproterenol-induced myocardial infarction $(37,38)$. This compound may thus prevent cardiovascular diseases. Therefore, HXHY herbs containing multiple compounds that target multiple proteins are expected to exhibit polypharmacological therapeutic effects, further allowing the prediction of new targets and applications for these herbs. These hypotheses are consistent with the principle of TCM, whereby diverse diseases are treated with the same herb.

In summary, findings from our study indicate that the compounds of HXHY herbs target multiple proteins associated with OA, which provides a molecular basis for the clinical application of HXHY herbs in multi-target therapeutic treatment of OA. Our findings also suggest that HXHY herbs may be used for the treatment of additional diseases besides OA. Moreover, the in silico approach adopted herein provides new insights on the molecular mechanisms underlying the beneficial effects of herbs used in TCM, thus promoting discovery of new drugs.

\section{Acknowledgements}

This study was supported by the Developmental Fund of ChenKeji Integrative Medicine (no. CKJ2010032).

\section{References}

1. Swoboda B: Epidemiological arthrosis research. Orthopade 30: 834-840, 2001 (In German).

2. Rubio-Terres C and Grupo del estudio VECTRA: An economic evaluation of chondroitin sulfate and non-steroidal anti-inflammatory drugs for the treatment of osteoarthritis. Data from the VECTRA study. Reumatol Clin 6: 187-195, 2010 (In Spanish).

3. Bitton R: The economic burden of osteoarthritis. Am J Manag Care 15 (Suppl 8): S230-S235, 2009.

4. Guo ZR: Drug promiscuity. Yao Xue Xue Bao 46: 361-369, 2011 (In Chinese).

5. Frantz S: Drug discovery: playing dirty. Nature 437: 942-943, 2005.

6. Ernst E: Complementary or alternative therapies for osteoarthritis. Nat Clin Pract Rheumatol 2: 74-80, 2006.

7. Rezende MU, Gurgel HM, Vilaca PR Jr, Kuroba RK, Lopes AS, Phillipi RZ and Hernandez AJ: Diacerhein versus glucosamine in a rat model of osteoarthritis. Clinics (Sao Paulo) 61: 461-466, 2006.

8. Choi JH, Choi JH, Kim DY, Yoon JH, Youn HY, Yi JB, Rhee HI, Ryu KH, Jung K, Han CK, et al: Effects of SKI 306X, a new herbal agent, on proteoglycan degradation in cartilage explant culture and collagenase-induced rabbit osteoarthritis model. Osteoarthritis Cartilage 10: 471-478, 2002.

9. Wang P, Gu EP and Cao HY: Effect of Huoxue Huayu plaster on NO and MMP-13 in joint fluid of osseous arthritis of knee in the early and middle stage. Tianjin Zhong Yi Yao 27: 192-193, 2010 (In Chinese)

10. Wang P, Gu EP and Cao HY: Clinical efficacy observation for osseous arthritis of knee in the early and middle stage treated with Huoxue Huayu plaster. Jilin Zhong Yi Yao 30: 491-492, 2010.

11. Liu XX, Li XH and Zhou JT: Research on mechanism of Tougu Xiaotong Granule in preventing and treating knee osteoarthritis. Zhongguo Zhong Xi Yi Jie He Za Zhi 27: 50-54, 2007 (In Chinese).

12. Yu F, Li X, Cai L, Li H, Chen J, Wong X, Xu H, Zheng C, Liu X and $\mathrm{Ye} \mathrm{H}$ : Achyranthes bidentata polysaccharides induce chondrocyte proliferation via the promotion of the $\mathrm{G}_{1} / \mathrm{S}$ cell cycle transition. Mol Med Rep 7: 935-940, 2013.

13. Zhu HF, Wang WJ and Wang ZM: Recent advances of Drynaria fortunei. Zhongguo Gu Shang 1: 66-68, 2009 (In Chinese).

14. Qiao X, Hou T, Zhang W, Guo S and Xu X: A 3D structure database of components from Chinese traditional medicinal herbs. J Chem Inf Comput Sci 42: 481-489, 2002.

15. Wishart DS, Knox C, Guo AC, Cheng D, Shrivastava S, Tzur D, Gautam B and Hassanali M: DrugBank: a knowledgebase for drugs, drug actions and drug targets. Nucleic Acids Res 36: D901-D906, 2008.
16. Dobson CM: Chemical space and biology. Nature 432: 824-828, 2004.

17. Huang Q, Qiao X and Xu X: Potential synergism and inhibitors to multiple target enzymes of Xuefu Zhuyu Decoction in cardiac disease therapeutics: a computational approach. Bioorg Med Chem Lett 17: 1779-1783, 2007.

18. Lipinski CA, Lombardo F, Dominy BW and Feeney PJ: Experimental and computational approaches to estimate solubility and permeability in drug discovery and development settings. Adv Drug Deliv Rev 46: 3-26, 2001.

19. Bonnet CS and Walsh DA: Osteoarthritis, angiogenesis and inflammation. Rheumatology (Oxford) 44: 7-16, 2005.

20. Loeser RF: Articular cartilage nuclear receptors: an emerging target for treatment of osteoarthritis. Osteoarthritis Cartilage 17: 829-831, 2009.

21. Tenor H, Hedbom E, Hauselmann HJ, Schudt C and Hatzelmann A: Phosphodiesterase isoenzyme families in human osteoarthritis chondrocytes - functional importance of phosphodiesterase 4. Br J Pharmacol 135: 609-618, 2002.

22. Montes M, Braud E, Miteva MA, Goddard ML, Mondesert O, Kolb S, Brun MP, Ducommun B, Garbay C and Villoutreix BO: Receptor-based virtual ligand screening for the identification of novel CDC25 phosphatase inhibitors. J Chem Inf Model 48: 157-165, 2008.

23. Zhu F, Shi Z, Qin C, Tao L, Liu X, Xu F, Zhang L, Song Y, Liu XH, Zhang J, et al: Therapeutic target database update 2012: a resource for facilitating target-oriented drug discovery. Nucleic Acids Res 40: D1128-D1136, 2012.

24. Smoot ME, Ono K, Ruscheinski J, Wang PL and Ideker T: Cytoscape 2.8: new features for data integration and network visualization. Bioinformatics 27: 431-432, 2011.

25. Assenov Y, Ramirez F, Schelhorn SE, Lengauer T and Albrecht M: Computing topological parameters of biological networks. Bioinformatics 24: 282-284, 2008.

26. Haringman JJ, Ludikhuize J and Tak PP: Chemokines in joint disease: the key to inflammation? Ann Rheum Dis 63: 1186-1194, 2004.

27. Wagner H: Multitarget therapy - the future of treatment for more than just functional dyspepsia. Phytomedicine 13 (Suppl 5): 122-129, 2006.

28. Espinoza-Fonseca LM: The benefits of the multi-target approach in drug design and discovery. Bioorg Med Chem 14: 896-897, 2006.

29. Jeong H, Mason SP, Barabasi AL and Oltvai ZN: Lethality and centrality in protein networks. Nature 411: 41-42, 2001.

30. Wang X, Zang B, Wu W, Tong J and Jin M: Protective effect of hydroxysafflor yellow A on endothelial cell injury induced by lipopolysaccharide. Zhongguo Zhong Yao Za Zhi 36: 1650-1653, 2011 (In Chinese).

31. Rosenbaum CC, O'Mathuna DP, Chavez M and Shields K: Antioxidants and antiinflammatory dietary supplements for osteoarthritis and rheumatoid arthritis. Altern Ther Health Med 16: 32-40, 2010.

32. Luo P, Tan Z, Zhang Z, Li H and Mo Z: Inhibitory effects of salvianolic acid $B$ on the high glucose-induced mesangial proliferation via NF- $\kappa \mathrm{B}$-dependent pathway. Biol Pharm Bull 31: 1381-1386, 2008.

33. Li AZ: The therapeutic effect of retinal vein occlusion with Huoxue Huayu method in traditional Chinese medicine. Guiding J Trad Chin Med Pharm 19: 63-65, 2013 (In Chinese).

34. Zhang P, Wang H and Ou YL: The therapeutic experience of Huoxue Huayu method in diabetic nephropathy. J Pract Tradit Chin Med 2: 130, 2013 (In Chinese).

35. Gao CX: Clinical practice of Huoxue Huayu method in eye diseases treatment. Seek medical and ask the medicine 10: 62, 2012 (In Chinese).

36. Liu H, Wang J, Zhou W, Wang Y and Yang L: Systems approaches and polypharmacology for drug discovery from herbal medicines: an example using licorice. J Ethnopharmacol 146: 773-793, 2013.

37. Tanabe H, Suzuki H, Nagatsu A, Mizukami H, Ogihara Y and Inoue M: Selective inhibition of vascular smooth muscle cell proliferation by coptisine isolated from Coptis rhizoma, one of the crude drugs composing Kampo medicines Unsei-in. Phytomedicine 13: 334-342, 2006.

38. Gong LL, Fang LH, Wang SB, Sun JL, Qin HL, Li XX, Wang SB and Du GH: Coptisine exert cardioprotective effect through anti-oxidative and inhibition of RhoA/Rho kinase pathway on isoproterenol-induced myocardial infarction in rats. Atherosclerosis 222: 50-58, 2012. 